\title{
REVIEW ARTICLE OPEN Magnetic structures and dynamics of multiferroic systems obtained with neutron scattering
}

\author{
William Ratcliff ${ }^{1}$, Jeffrey W Lynn ${ }^{1}$, Valery Kiryukhin ${ }^{2}$, Prashant Jain $^{3}$ and Michael R Fitzsimmons ${ }^{3}$
}

Multiferroics are materials that evince both ferroelectric and magnetic order parameters. These order parameters when coupled can lead to both exciting new physics as well as new device applications. Potential device applications include memory, magnetic field sensors, small antennas and so on. Since Kimura's discovery of multiferroicity in $\mathrm{TbMnO}_{3}$, there has been a renaissance in the study of these materials. Great progress has been made in both materials discovery and in the theoretical understanding of these materials. In type-II systems the magnetic order breaks the inversion symmetry of the material, driving a secondary ferroelectric phase transition in which the ferroelectric polarisation is exquisitely coupled to the magnetic structure and thus to magnetic field. In type-I systems, the magnetic and ferroelectric orders are established on different sublattices of the material and typically are weakly coupled, but electric field can still drive changes in the magnetisation. Besides single-phase multiferroics, there has been exciting progress in composite heterostructures of multiferroics. Here, we review neutron measurements of prototypical examples of these different approaches to achieving multiferrocity.

npj Quantum Materials (2016) 1, 16003; doi:10.1038/npjquantmats.2016.3; published online 27 July 2016

\section{INTRODUCTION}

Neutron scattering plays an important role in determining the ferroelectric properties of multiferroics in terms of the detailed crystal structure, but the central role is in elucidating the magnetic structures and spin dynamics, and in understanding the origin of how, and how strongly, the magnetic and ferroelectric order parameters are coupled. We first review very briefly the key neutron techniques employed to investigate multiferroics, both in bulk and thin-film forms. We emphasise the role of magnetic scattering here but also reference the standard techniques for crystal structure refinements and exploring the lattice dynamics, which are similar in concept to magnetic Bragg scattering and measuring the spin dynamics, respectively. We then discuss two systems which are prototypes for a type-I multiferroic material $\left(\mathrm{HoMnO}_{3}\right)$, and a type-II multiferroic material $\left(\mathrm{TbMnO}_{3}\right)$. The type I ferroelectrics typically have high-ferroelectric transition temperatures-well above room temperature-but low magnetic ordering temperatures, and these two disparate order parameters are weakly coupled. The type II ferroelectrics are typically materials with low magnetic ordering temperatures, where the magnetic structure itself breaks the inversion symmetry and permits (typically very weak) ferroelectric order to develop. Both are interesting from a fundamental point of view, but so far only some of the type I materials exhibit both types of orders at room temperature and thus appear to have promise for applications such as memory, spintronics and sensors. One such type I multiferroic is $\mathrm{BiFeO}_{3}$, which is the leading candidate material for applications, and we discuss the results for bulk crystals and thin films. We then explore the properties of nanocomposites and hybrid inorganic-organic materials, and conclude with prospects for further work.

\section{DIFFRACTION (STRUCTURE) AND INELASTIC SCATTERING (DYNAMICS)}

Neutron scattering have a central role in determining the crystal and magnetic structures of a vast variety of materials. One traditional role in the magnetically ordered regime has been the measurement of magnetic Bragg intensities, which can be used to determine the fundamental nature and symmetry of the magnetic state. Quantitative values of the ordered moment(s) as a function of temperature, pressure and applied magnetic field can be determined, as well as the density of unpaired electrons that constitute the magnetic moments. These are elastic scattering measurements (no change in the energy of the neutrons) which are carried out on single crystals, powders, thin films and multilayers. For thin films and multilayers the specialized elastic scattering technique of neutron reflectometry is employed, which can determine both the atomic and magnetic density depth profiles averaged over the area of the film. For large scale structures $(1-1,000 \mathrm{~nm})$ the technique of (elastic) small-angle neutron scattering (SANS) is used. Neutron diffraction studies often provide information that can be obtained by no other experimental technique. In the investigation of the spin dynamics of systems, e.g., neutrons play a truly unique role. Neutron scattering is the only technique that can directly determine the complete magnetic excitation spectrum, whether it is in the form of the dispersion relations for spin wave excitations, wave vector and energy dependence of critical fluctuations, crystal field excitations, moment fluctuations and so on, which can be readily compared with theory. Techniques employing the spin-dependent scattering of the neutron can be used to unambiguously identify nuclear scattering from magnetic scattering, both for magnetic/ crystal structures and for excitations. More detailed reviews of neutron magnetic scattering techniques, including the theory of

${ }^{1}$ NIST Center for Neutron Research, National Institute of Standards and Technology, Gaithersburg, MD, USA; ${ }^{2}$ Department of Physics and Astronomy, Rutgers University, Piscataway, NJ, USA and ${ }^{3}$ Los Alamos National Laboratory, Los Alamos, NM, USA.

Correspondence: W Ratcliff (william.ratcliff@nist.gov)

Received 6 May 2016; revised 8 June 2016; accepted 9 June 2016 
neutron scattering from magnetic systems and solids, can be found in references $1-5$.

\section{POLARISED NEUTRON REFLECTOMETRY (PNR)}

PNR is ideally suited to measure the nuclear and magnetisation depth profiles across planar interfaces. Because reflection only occurs when one or both of the nuclear or magnetic compositions change across an interface, PNR can distinguish magnetism at interfaces and other nanoscale structures from contamination within a substrate or on the sample. ${ }^{6}$ Extensive reviews of PNR can be found in references 7-9.

Briefly, in PNR the intensity of the specularly reflected neutron beam is compared with the intensity of the incident beam as a function of wavevector transfer, $Q(=4 \pi \sin \theta / \lambda$, where, $\theta$ is angle of incidence and $\lambda$ is the neutron wavelength), and neutron beam polarisation (Figure 1a). $Q$ is changed by changing $\theta$ (typically of order $1^{\circ}$ ) or $\lambda$ (typically of order $0.2-1.4 \mathrm{~nm}$ ). The specular reflectivity, $R$, is determined by the neutron scattering length density (SLD) depth profile, $\rho(z)$, averaged over the lateral dimensions of the sample. The SLD is the depth-dependent variation of the index of refraction (related to $\rho(z)$ ) of the sample.
Although the reflectivity close to the origin is highly nonkinematical, the reflectivity at larger $Q$ can be thought of in terms of the square of the modulus of the Fourier transform of the SLD profile. (Widely available numerical codes permit rigorous analysis including the non-kinematical behaviour of the reflectivity for all Q.) $\rho(z)$ consists of nuclear and magnetic SLDs such that $\rho^{ \pm}(z)=\rho_{n}(z) \pm C M(z)$, where $C=2.9109 \times 10^{-9} \AA^{-2}(\mathrm{kA} / \mathrm{m})^{-1}$, and $M(z)$ is the magnetisation $(\mathrm{kA} / \mathrm{m})$ depth profile., ${ }^{8,9}$ Unlike magnetometry, which measures magnetic moment, reflection of polarised neutron beams depends on a variation of moment density across a planar interface. Further, unlike non-resonant $X$-ray scattering, the nuclear and magnetic neutron scattering lengths have comparable magnitudes. The $+(-)$ sign in $\rho^{ \pm}(z)$ denotes neutron beam polarisation parallel (opposite) to the applied magnetic field and corresponds to spin-dependent reflectivities, $R^{ \pm}(Q)$. By measuring $R^{+}(Q)$ and $R^{-}(Q), \rho_{n}(z)$ and $M(z)$ can be obtained separately. If the net magnetisation is rotated away from the applied field, polarisation analysis of the specularly reflected beam provides information about the projection of the net magnetisation vector onto the sample plane. For example, polarisation analysis with PNR is useful for identifying the magnetic orientations of different layers parallel to the sample plane.

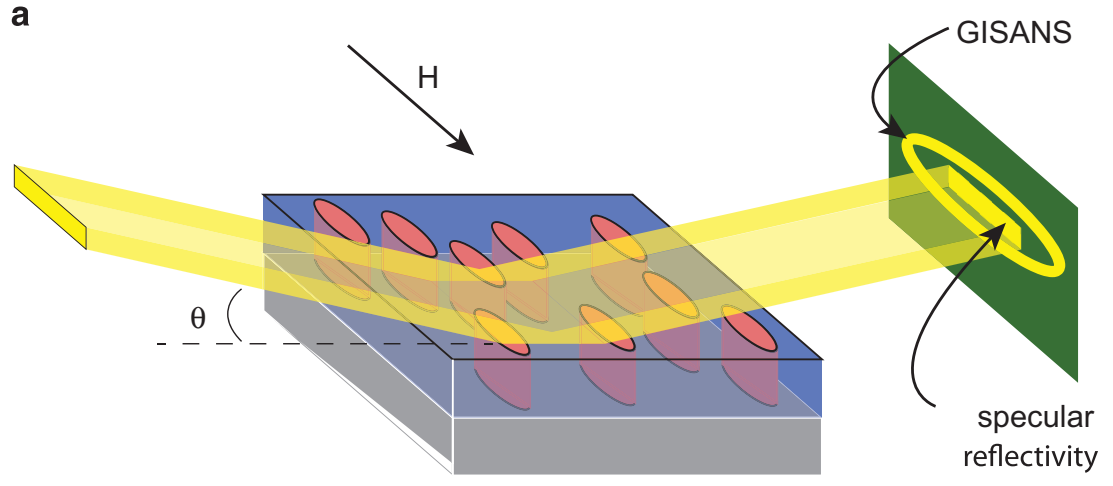

b
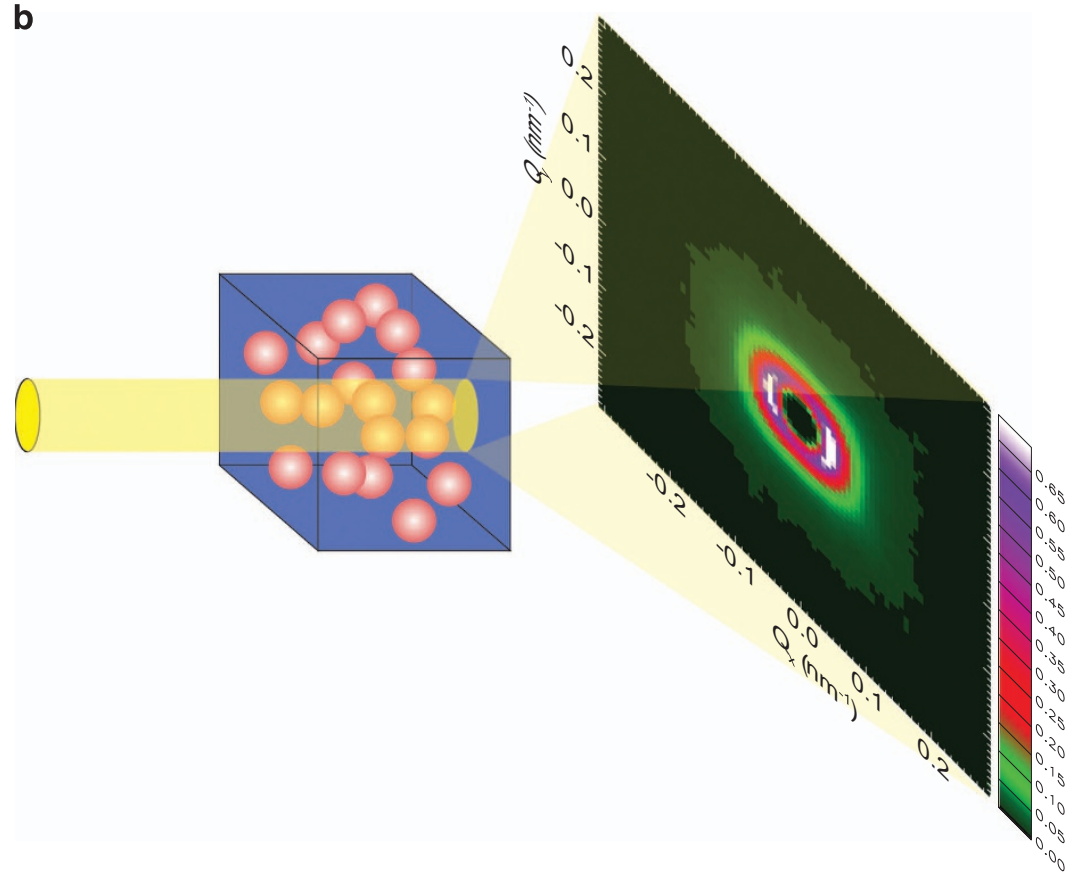

Figure 1. (a) Schematic of a reflectometry experiment with the possibility of off-specular scattering (schematically labelled as GISANS for grazing incidence small angle scattering) about the specularly reflected beam. The applied magnetic field is shown by $\mathrm{H}$. (b) A SANS experiment. 


\section{SMALL-ANGLE NEUTRON SCATTERING}

SANS is ideally suited to measure the change of nuclear and magnetisation contrast across non-planar interfaces. In SANS experiments the incident neutron beam is tightly collimated in two orthogonal directions (Figure 1b). SANS provides information about regions, domains and particles bounded by interfaces

a<smiles>CC(=O)P</smiles>

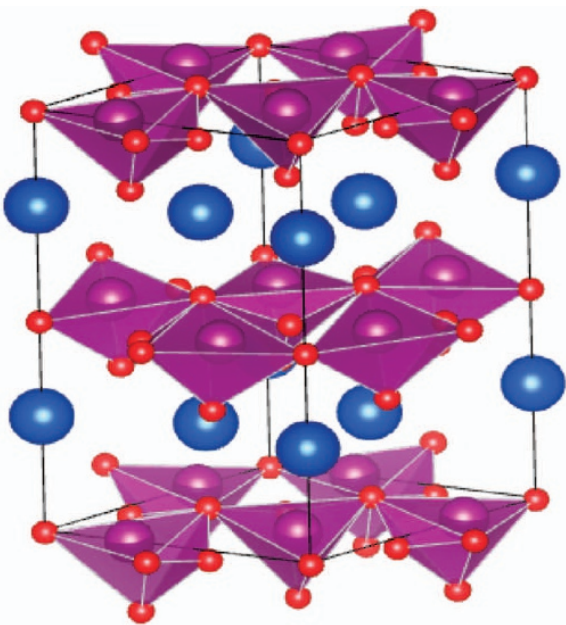

b

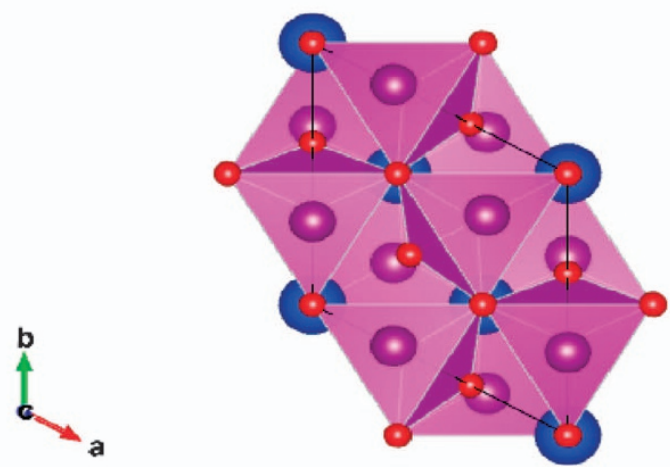

Figure 2. Crystal structure for hexagonal $\mathrm{HoMnO}_{3}$. (a) View from the side ( $c$ axis is up). Large blue balls are Ho, red balls are oxygen. The $\mathrm{Mn}$ ions sit at the centre of each tetrahedron of oxygen ions. (b) View along the $c$ axis. Note the triangular configuration of the $\mathrm{Mn}$ (oxygen tetrahedra) as well as the Ho. The oxygen that make up the sides of each tetrahedron also exhibit triangular coordination. across which either the nuclear or magnetic SLD changes. The length scales probed by SANS vary between a few nanometres and microns. Because of the inverse relationship between length scale and reciprocal space, short-length scales produce SANS furthest from the primary (unscattered) beam. Like PNR, polarised SANS allows an unambiguous identification of the nuclear and magnetic scattering and the relationship between the two.

Confinement of scattered intensity in neutron reflectometry about the specular reflectivity allows the use of supermirrors for polarisation analysis with relative ease. However, polarisation analysis of widely divergent SANS with mirrors is more challenging. The availability of $\mathrm{He}^{3}$ polarising filters has recently enabled routine polarisation analysis for SANS, allowing successful exploitation of SANS for the unique identification of magnetic and structural contributions to the scattering from magnetic materials. ${ }^{10-12}$ In some cases neutron scattering with polarisation analysis enables measurement of correlations of magnetism that transcend the physical non-uniformity of the material. ${ }^{13}$

Sample environments for neutron scattering experiments routinely include cryomagnets providing fields as large as $15 \mathrm{~T}$, temperatures as low as $20 \mathrm{mK}$, pressure cells, mechanical devices (to apply bending stress to substrates) and facilities for irradiation with light. Equipment exists that offers opportunities to simultaneously control magnetic/electric fields, temperature and pressure-of obvious interest to studies of magnetoelectric multiferroic materials.

\section{TYPE-I MULTIFERROIC: $\mathrm{HOMNO}_{3}$}

The hexagonal (space group $\mathrm{P}_{3} \mathrm{~cm}$ ) rare-earth manganese oxides $\left(R \mathrm{MnO}_{3}\right.$ with $R=\mathrm{Ho}, \mathrm{Er}, \mathrm{Tm}, \mathrm{Yb}, \mathrm{Lu}$ and $\left.\mathrm{Y}\right)$ are a family of multiferroic materials of particular interest, and $\mathrm{HoMnO}_{3}$ is a prototypical system representative of these hexagonal materials that has been investigated in considerable detail. In this material the Ho-O ions undergo a displacement at very high temperature $\left(T_{C}=1375 \mathrm{~K}\right){ }^{14}$ which gives rise to a ferroelectric (FE) moment along the $c$ axis. Long-range magnetic order, on the other hand, develops for the $\mathrm{Mn}$ moments at $72 \mathrm{~K}$. The disparate ordering temperatures indicate that this system is a type-I multiferroic where the order parameters are relatively weakly coupled through the Ho-Mn exchange and anisotropy interactions. The magnetic system has the added interest that the Mn moments occupy a frustrated triangular lattice as shown in Figure 2. Below the magnetic ordering temperature $\left(T_{\mathrm{N}}=72 \mathrm{~K}\right)$ the $\mathrm{Mn}$ spins order in a noncollinear $120^{\circ}$ spin structure. At $40 \mathrm{~K}$ a spin reorientation $\left(T_{\mathrm{SR}}=40 \mathrm{~K}\right)$ takes place in which the $\mathrm{Mn}$ moments rotate in the
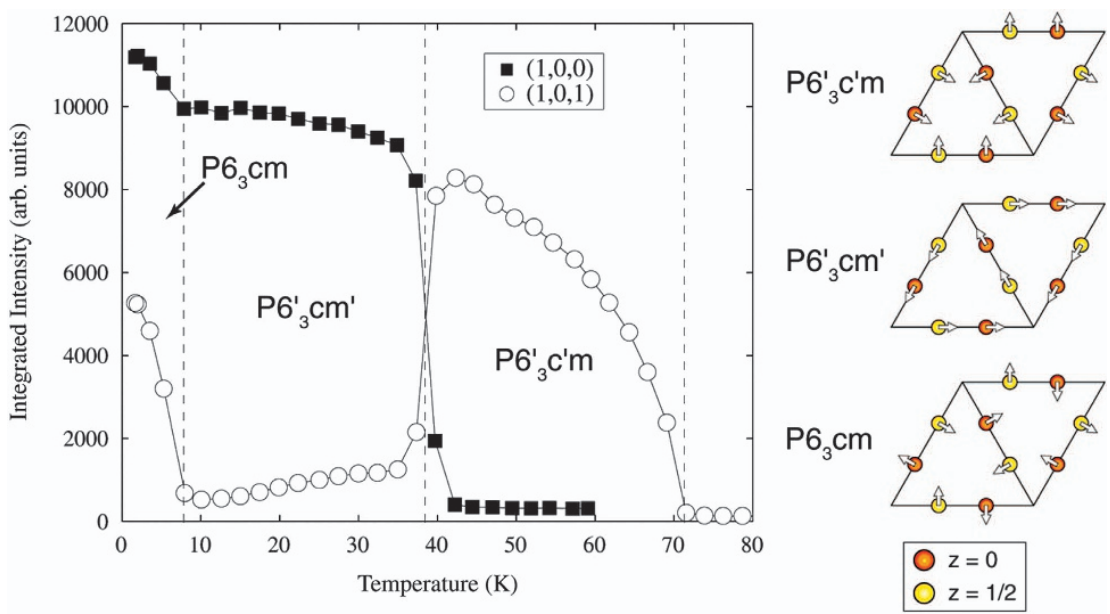

Figure 3. Neutron diffraction measurements of the integrated intensities of the (100) and (101) magnetic Bragg reflections of $\mathrm{HoMnO}_{3}$. Two spin-reorientation transitions (indicated by dashed lines) lead to changes in the intensities of both peaks. The Mn ${ }^{3+}$ spin configurations for these phases are shown on the right. $\mathrm{Ho}^{3+}$ moments (not shown) order in the low-temperature phase. (Adapted from reference 17.) 


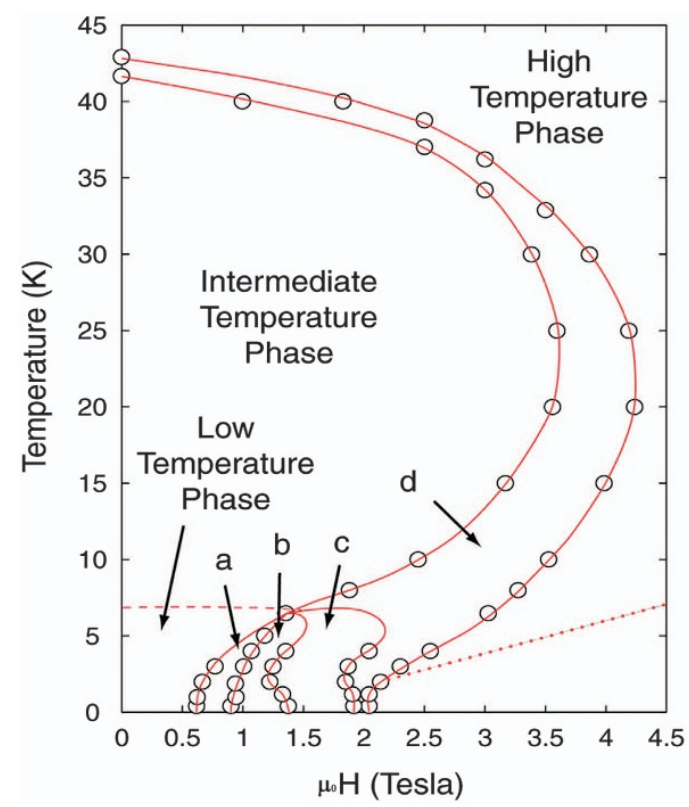

Figure 4. Temperature versus magnetic field phase diagram for $\mathrm{HoMnO}_{3}$ obtained from neutron diffraction measurements. Curves are guides-to-the-eye. The dashed line and dotted lines indicate approximate phase boundaries for previously reported transitions not observable from the diffraction data. The numbered regions correspond to the intermediate phases and hysteretic overlap regions that occur at low T. (Adapted from reference 17.) plane, changing the magnetic symmetry and adopting a different $120^{\circ}$ spin structure. At low temperatures the Ho moments order antiferromagnetically $\left(T_{\mathrm{Ho}}=8 \mathrm{~K}\right)$ with moments aligned along the $C$ axis, accompanied by a second spin reorientation transition of the $\mathrm{Mn}$ moments into the $P 63 \mathrm{~cm} 120^{\circ}$ spin structure phase. The spin structures in these phases are shown in Figure 3. The sharp magnetic transitions shown in the figure are accompanied by sharp anomalies in the dielectric constant, indicating that the magnetic and ferroelectric order parameters are coupled. ${ }^{15}$ Second-harmonic generation measurements as a function of magnetic field also show the change in magnetic symmetry and the reentrant phase as a function of magnetic field suggested by dielectric susceptibility. ${ }^{16}$

The application of a magnetic field shifts $T_{\mathrm{SR}}$ to lower $T$, broadens the transition, and drives $\mathrm{HoMnO}_{3}$ into a reentrant phase as shown in the ( $\mathrm{H}$ versus $\mathrm{T}$ ) phase diagram of Figure $4 .^{2}$ In the intermediate-temperature phase, a sufficiently strong applied magnetic field along the $c$ axis pushes $\mathrm{HoMnO}_{3}$ into the high-temperature phase, ${ }^{15-17}$ in good agreement with the phase diagram established from dielectric susceptibility measurements. ${ }^{15-18}$ At low temperatures, as ordered Ho magnetic order develops, the phase diagram becomes considerably more complicated, including a balancing of the interactions to produce a critical end point around $2 \mathrm{~T}$ and $2 \mathrm{~K}^{18}{ }^{18}$ indicative of the rich physics of $\mathrm{HoMnO}_{3}$. In this temperature regime it is expected that the magnetic and ferroelectric order parameters naturally couple since the Ho moments are magnetically ordered while the ferroelectric order originates with the Ho-O displacements.

The spin dynamics of this non-collinear frustrated triangular magnetic lattice has been investigated in considerable detail.

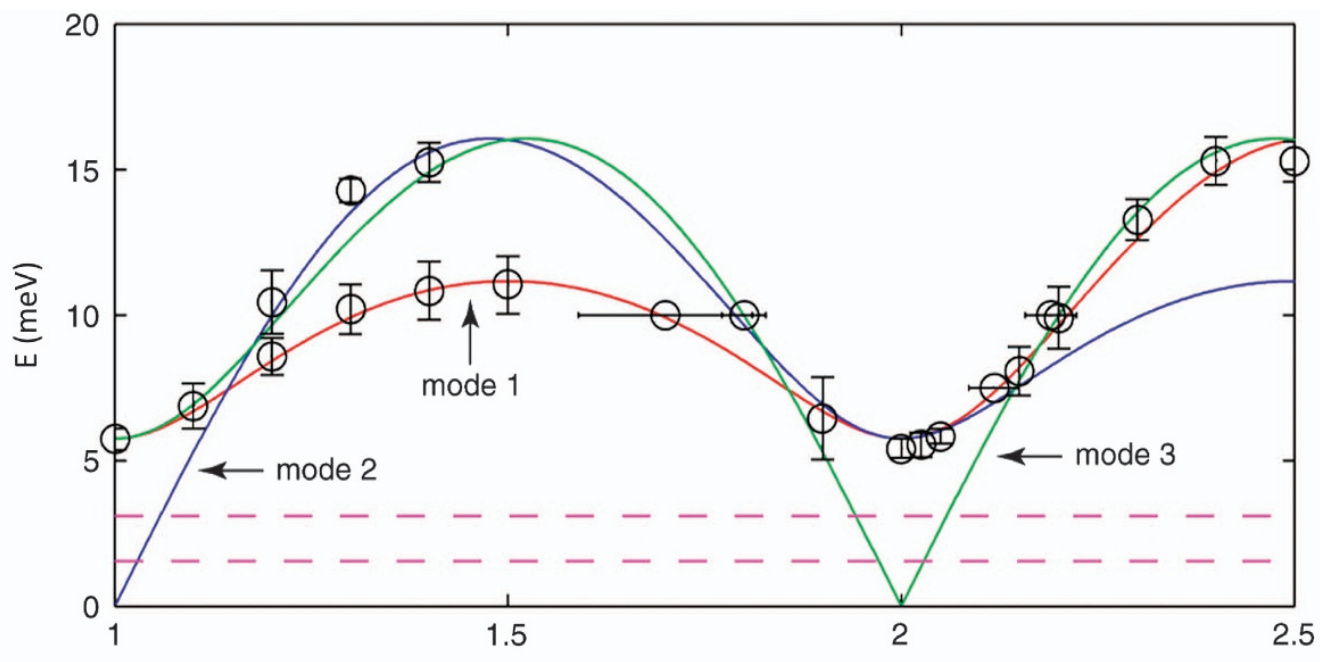

$(\mathrm{H}, 0,0)$
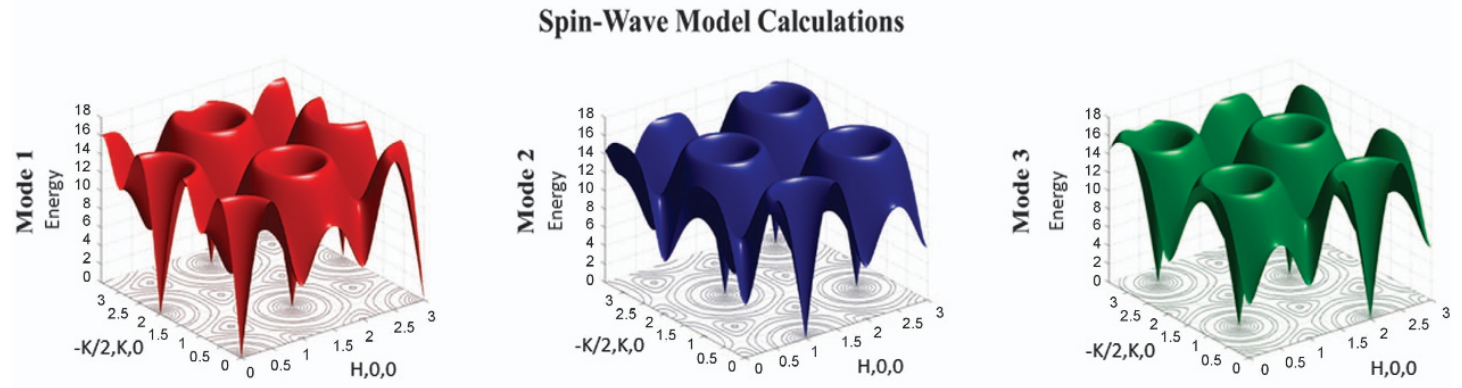

Figure 5. In-plane spin-wave dispersion for $\mathrm{HoMnO}_{3}$ at $20 \mathrm{~K}$ for the three modes. Dashed lines indicate two (dispersionless) crystal field levels of Ho at 1.5(1) and 3.1(1) meV. The bottom part of the figure shows the calculated spin wave dispersion relations for the three modes. The dispersion curves for the three modes are identical, but the origins are offset for the three. (Adapted from reference 17.) 
a

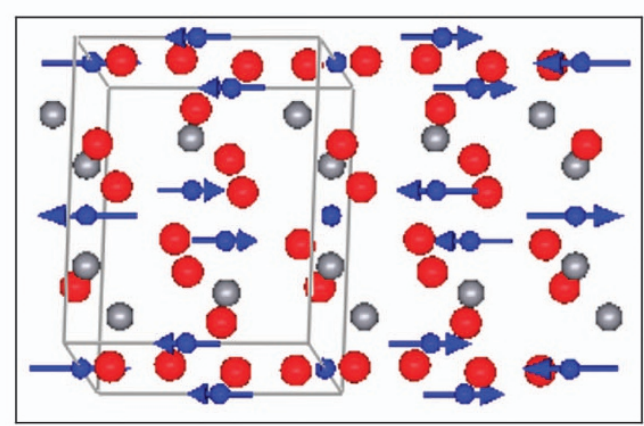

C

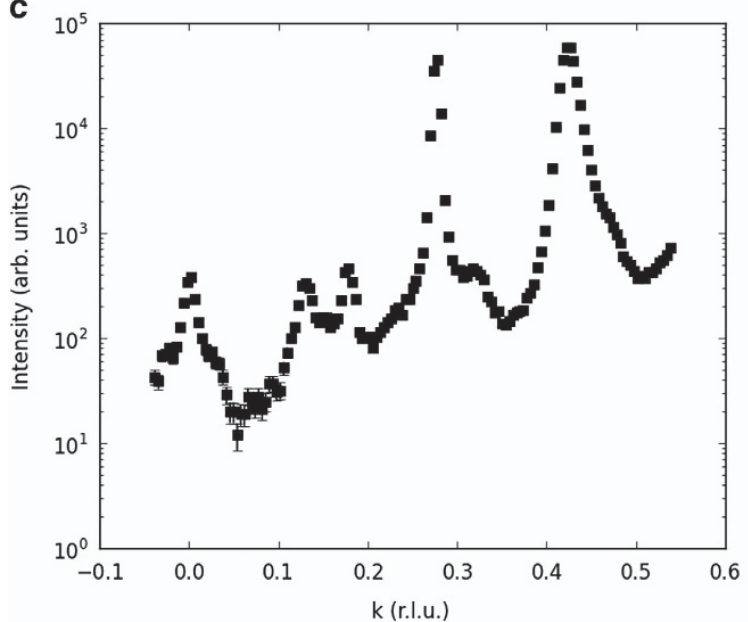

b

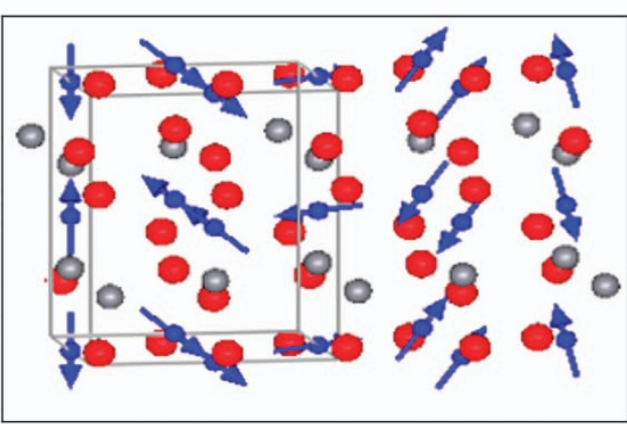

d

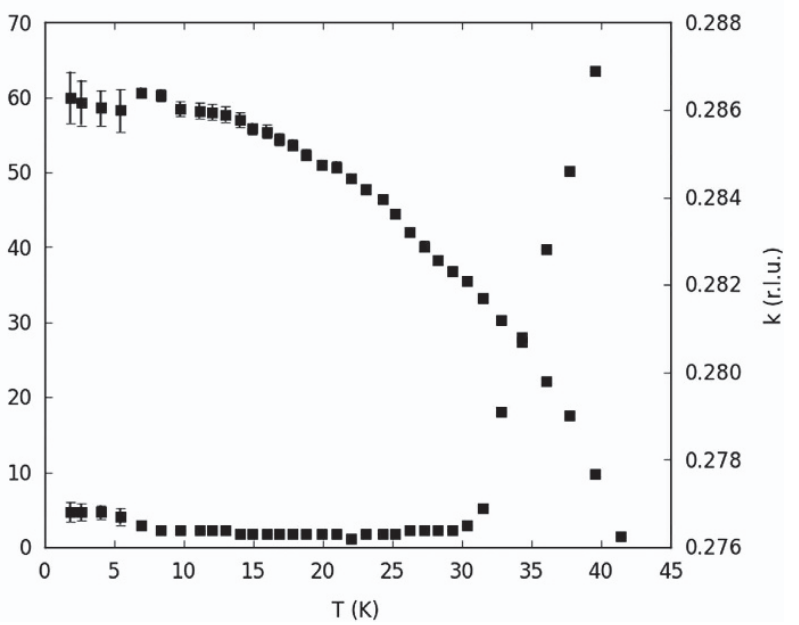

Figure 6. (a) Cartoon of the SDW magnetic structure above $28 \mathrm{~K}$ for $\mathrm{TbMnO}_{3}$. Moments are only shown on the Mn ions. (b) Cartoon of magnetic structure below $28 \mathrm{~K}$ in the elliptical spiral phase. Moments are only shown on the Mn ions. (c) Example magnetic diffraction pattern along a high symmetry direction at $2 \mathrm{~K}$, with the intensity on a log scale. (d) Temperature dependence of the intensity, which is proportional to the square of the order parameter, and the incommensurate position $k$, of the (0k1) reflection versus temperature. Arrows indicate with which axis symbols are associated. (Adapted from reference 24.)

It turns out that the coupling along the $c$ axis is very weak, so the spin dynamics is two-dimensional in nature. A Hamiltonian that captures the basic physics of the problem can be written as

$$
H=-\sum_{i, j} J_{i j} \overrightarrow{S_{i}} \cdot \overrightarrow{S_{j}}-D \sum_{i}\left(S^{z}{ }_{i}\right)^{2}
$$

where $J_{i j}=J$ is the strength of the nearest-neighbour in-plane antiferromagnetic (AFM) exchange interaction and $D$ is a singleion anisotropy. The spin-wave spectrum then consists of three separate modes that propagate within the hexagonal plane, which are equivalent in energy but their origins are offset in wave vector. ${ }^{17,19}$ Figure 5 shows a comparison of the model with the data taken at $20 \mathrm{~K}$ (intermediate temperature phase) along the high-symmetry directions in reciprocal space, using $J$ and $D$ as fitting parameters. From these fits we find $J=2.44 \mathrm{meV}$ and $D=0.38 \mathrm{meV}$. The anisotropy was found to have a significant temperature dependence, which is the driving force behind the spin reorientation transitions observed in this system. We note that such spin reorientations do not occur in $\mathrm{YMnO}_{3}$, so it is clear that they originate from the holmium, as has been observed in other rare earth systems such as $\mathrm{Nd}_{2} \mathrm{CuO}_{4}{ }^{20}$ We note that the full-magnetic Hamiltonian for $\mathrm{HoMnO}_{3}$ including the Ho moments and ferroelectric coupling must be considerably more complex than Equation (1), but this very simple model establishes the dominant magnetic interactions for this system.

\section{TYPE-II MULTIFERROIC: $\mathrm{TBMNO}_{3}$}

The orthorhombic (space group $\mathrm{Pbnm}$ ) $\mathrm{RMnO}_{3}$ series of perovskite manganites, where $R=[\mathrm{Gd}, \mathrm{Tb}, \mathrm{Dy}]$, show a variety of magnetic orderings depending on the size of the $R$ ion. ${ }^{21}$ The $\mathrm{Mn}^{3+}$ ions lie on four Bravais lattices at the: $1(1 / 200), 2\left(\begin{array}{lll}1 / 2 & 0 & 1 / 2\end{array}\right), 3\left(\begin{array}{lll}0 & 1 / 2 & 1 / 2\end{array}\right)$ and $4\left(\begin{array}{lll}0 & 1 / 2 & 0\end{array}\right)$ positions. These $\mathrm{Mn}^{3+}$ ions have an electronic configuration of $3 t_{2 g} e_{g}$ with the nearest neighbour $t_{2 g}$ electrons having AFM couplings and the $e_{g}$ electrons having ferromagnetic coupling. ${ }^{21}$ Competition between these couplings gives rise to a variety of $\mathrm{Mn}$ magnetic orderings, ranging from the simple antiferromagnetism of $\mathrm{LaMnO}_{3}$ to a complex incommensurate ordering in $\mathrm{TbMnO}_{3}$. In addition to $\mathrm{TbMnO}_{3}$ ordering magnetically at $41 \mathrm{~K}$, at $\sim 28 \mathrm{~K}$ a spontaneous ferroelectric polarisation develops. ${ }^{22-24}$ This polarisation can be switched at low temperatures through the application of a strong magnetic field. ${ }^{22}$

Initial studies ${ }^{23}$ of the magnetic order of $\mathrm{TbMnO}_{3}$ were able to identify the ordering wave-vector to be along the $b$ axis and to determine that it was incommensurate and the incommensurability changed with temperature. A symmetry analysis for the $30 \mathrm{~K}$ data indicated the presence of only one Fourier component for the incommensurate wave vector, with the spins obeying either an amplitude modulation or a spiral structure. On model refinement to the data, it was found that the moment was along the $b$ axis and the magnetic structure was amplitude modulated. Thus the initial ordering of the Mn spins is in the form of a simple longitudinally polarised spin-density wave (SDW).

Subsequent data are shown in Figure 6, taken by Kenzelmann et $a l_{.}{ }^{24}$ who performed a more detailed examination of the system, in particular to elucidate the relationship between the magnetic structure and ferroelectricity. They used representational analysis to classify the magnetic structures possible with the underlying space group symmetry. They found that there were 
four representations (i.e., families of magnetic structures) consistent with the space group symmetry and ordering wave-vector. At high temperatures, the system orders with a single representation and is consistent with previous results. However, below $28 \mathrm{~K}$ two representations are required to describe the magnetic ordering. In this phase, the Mn moments form an elliptical spiral, which breaks the symmetry and permits ferroelectric order to develop. The magnetic structure is shown in Figure $6 \mathrm{~b}$. Later magnetic X-ray scattering measurements further refined this picture, however, the basic physics remains the same. ${ }^{25}$ There have been recent measurements using neutron diffraction to examine the effects of pressure and electric fields on the magnetic ordering in these materials. ${ }^{26,27}$

Kenzelmann et $a l^{24}$ showed that the electric polarisation observed in this system can be explained by a trilinear coupling term in the free energy. By symmetry, this term is necessarily absent in the simple spin density phase, but is allowed in the phase below $28 \mathrm{~K}$ when the spiral component of the magnetic order develops. This term allows ferroelectric polarisation along the $c$ axis, as observed. Another phenomenological model was developed by Mostovoy ${ }^{28}$ in which the induced polarisation is dependent on a gradient in the magnetisation, while a microscopic model based on an inverse Dzyaloshinskii-Moriya (DM) coupling was developed by Katsura et al. ${ }^{29}$ in which the spontaneous ferroelectric polarisation which arises in the spiral phase is given by: $\vec{P} \propto \overrightarrow{r_{i j}} \times\left(\overrightarrow{S_{i}} \times \overrightarrow{S_{j}}\right)$. In this latter model the ferroelectric polarisation is along the direction perpendicular to both the spiral axis and the vector separating the moments, $\mathbf{r}_{\mathrm{ij}}$, and is consistent with that observed in $\mathrm{TbMnO}_{3}$. Other microscopic theoretical approaches have included $a b$ initio calculations, Monte Carlo simulations, and electric current cancellation. $^{30-33}$

In this system, the magnetic structure results from competing interactions, and neutron scattering can be used to probe these interactions through measurements of the spin wave spectrum. The same Hamiltonian (Equation (1)) ${ }^{34,35}$ as used for $\mathrm{HoMnO}_{3}$ can be employed with a nearest neighbour ( $a b$-plane) ferromagnetic exchange, $J_{F M}$, and a next-nearest-neighbour AFM interaction $J_{\text {AFM }}$ between planes, along with a single-ion anisotropy. Simple fits to this model yield $J_{\mathrm{FM}} \sim 0.15 \mathrm{meV}, J_{\mathrm{AFM}} \sim-0.5 \mathrm{meV}$ and D 0.13 meV. $^{35}$

However, there are interesting excitations beyond simple spin waves. One interesting mode is a 'phason' mode in which the phase of the moments in the spiral is changed, but the overall spin structure is invariant. Without anisotropy this would be a
Goldstone mode, ${ }^{36}$ however, it is shifted to finite energies (gapped) due to the anisotropy in the system. ${ }^{35}$ Beyond this, if we consider time-dependent fluctuations of the magnetic moments, these fluctuations will necessarily result in fluctuations in the $\overrightarrow{S_{i}} \times \overrightarrow{S_{j}}$ term in the induced ferroelectric polarisation. ${ }^{35}$ There is one mode which rotates the spin plane around the $c$ axis and another which rotates it around the $b$ axis. The latter rotation $^{35}$ will cause a fluctuating polarisation along the $a$ axis. We call this hybridised mode an 'electromagnon', and it has been observed by polarised inelastic neutron scattering. The electromagnon occurs at energies consistent with modes observed in optical measurements. ${ }^{37}$

Although there is some excitement in seeing that the ferroelectric polarisation in this system arises from the magnetic order, there is even more excitement in finding that the magnetic order can be controlled by an electric field as was done for $\mathrm{Ni}_{3} \mathrm{~V}_{2} \mathrm{O}_{8}{ }^{38}$ and that the ferroelectric polarisation can be controlled by magnetic fields. In $\mathrm{TbMnO}_{3}$ application of a strong magnetic field along either $\boldsymbol{a}$ or $\boldsymbol{b}$ axes causes the electric polarisation to flop. Speculation that the flop of the ferroelectric polarisation was caused by a flop of the plane of the spiral was confirmed by examining the field dependence of the spin wave spectrum. ${ }^{39}$ Further, application of a high-magnetic field changes the commensurability of the magnetic order.

\section{ROOM TEMPERATURE MULTIFERROIC: BIFEO $_{3}$}

$\mathrm{BiFeO}_{3}$, known as bismuth ferrite or BFO, occupies a special place in the list of known multiferroics. Both the ferroelectric $\left(T_{C} \sim 1,150 \mathrm{~K}\right)$ and magnetic $\left(T_{\mathrm{N}} \sim 640 \mathrm{~K}\right)$ transition temperatures are much higher than room temperature, and the FE polarisation $\left(\sim 90 \mu \mathrm{C} / \mathrm{cm}^{2}\right)$ exceeds the values exhibited by prototypical perovskite ferroelectrics such as $\mathrm{PbTiO}_{3}{ }^{40}$ The promise for engineering applications associated with these properties, as well as scientific interest, has motivated significant research activity. ${ }^{40}$ Although BFO has been known since the 1950 's, ${ }^{41}$ large single crystals have only become available recently. ${ }^{42,43}$ Lack of highquality single crystals impeded early neutron studies of BFO, consequently, several quantities of basic importance, such as the detailed magnetic structure and microscopic magnetic interactions, remained unknown until the late 2000's.

BFO exhibits a rhombohedral $R 3 C$ perovskite structure which will be described here using a pseudo-cubic notation with $a=3.96 \AA$, and $a \sim 89.4^{\circ}$. The electric polarisation points along the elongated body diagonal-the [111] direction. ${ }^{40}$ The magnetic

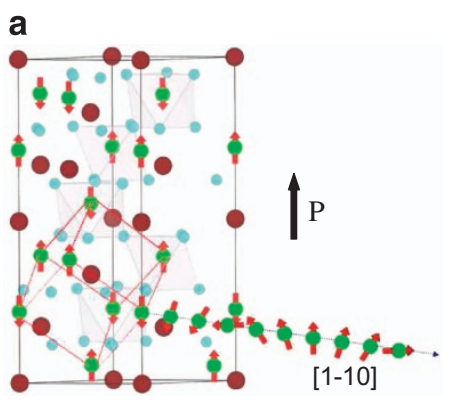

b
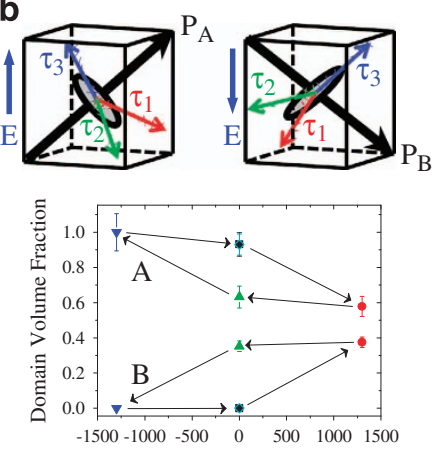

$\mathrm{E}(\mathrm{kV} / \mathrm{m})$

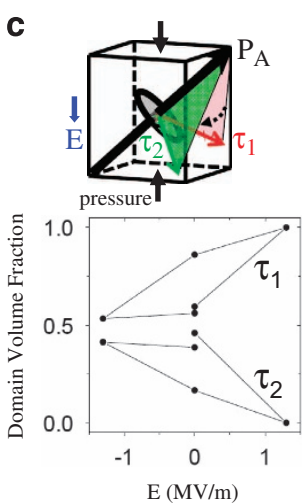

Figure 7. (a) Lattice and magnetic structures of $\mathrm{BiFeO}_{3}$ (adapted from reference 47). Red lines show the perovskite pseudo-cubic cell. (b) Top. Applied electric field E switches ferroelectric domain A into domain B. The magnetic propagation vectors $\tau_{\mathrm{i}}$ rotate together with the electric polarisation vector P. Bottom. Volume fractions of the domains A and B in an applied field. Both the magnetic signal ( $\tau_{i}$ wave vectors) and the structural signal (due to the elongated [111] diagonal) give essentially the same dependence. (Adapted from reference 43) (c) Within a single-ferroelectric domain, applied electric field or uniaxial pressure convert one equivalent magnetic domain $\left(\tau_{\mathrm{i}}\right)$ into another. Dashed arrow shows corresponding rotation of the magnetic easy plane. Bottom. Volume fractions of the domains $\tau_{1}$ and $\tau_{2}$ in an electric field. (No domain $\tau_{3}$ was detected in this sample; adapted from references 46,50 .) 
structure has long been known to be of the AFM G type (each nearest neighbour spin antiparallel), with a long period $(62 \mathrm{~nm})$ cycloidal modulation superimposed. ${ }^{44}$ The modulation can propagate along three symmetry-equivalent propagation vectors $\tau_{1}=\delta[1,-1,0], \tau_{2}=\delta[1,0,-1]$, and $\tau_{3}=\delta[0,-1,1]$, all normal to the electric polarisation, where $\delta \sim 0.0045$ reciprocal lattice units. ${ }^{43-46}$ In each of the three possible magnetic domains, the spins rotate in the plane defined by the polarisation vector [111], and $\tau$. The crystal and magnetic structures of BFO are depicted in Figure $7 a^{47}$

Both the ferroelectric vector and the magnetic easy plane of each of the magnetic domains are defined by the crystallographic lattice, leading to an effective magnetoelectric coupling. In this chapter, we consider two types of magnetoelectric coupling that allow control of the magnetic order by an applied electric field (a subject of special interest in multiferroics research). One type involves switching of the electric polarisation and the other does not. Both types originate from the magnetic anisotropy defining the plane of the cycloid. Figure $7 \mathrm{~b}$ illustrates the first effect. As the electric polarisation rotates ('switches') in an applied electric field, so do the magnetic easy planes defined by the ferroelectric vector and the directions of $\tau_{\mathrm{i}}$ that stay normal to this vector. In neutron diffraction studies of single crystals, the volume fractions of the different ferroelectric domains, as well as those of the magnetic domains coupled to them, can be directly inferred from the intensities of the appropriate Bragg peaks. ${ }^{43,45,46} \mathrm{~A}$ practical importance of magnetoelectric coupling was demonstrated in thin films of BFO covered by a ferromagnetic Co/Fe layer. ${ }^{48}$ The magnetic moment of the $\mathrm{Co} / \mathrm{Fe}$ layer was coupled to the easy plane position of the underlying BFO. As an electric field was applied, causing the ferroelectric polarisation to rotate, the magnetisation of the $\mathrm{Co} / \mathrm{Fe}$ layer also rotated, demonstrating control of a ferromagnetic moment by an electric field in a room-temperature device (composite multiferroics are discussed later in this chapter).

The second magnetoelectric effect revealed in neutron diffraction experiments does not involve ferroelectric switching and occurs within a single-ferroelectric domain. As an electric field is applied, the populations of the three equivalent magnetic domains defined by the vectors $\tau_{1}, \tau_{2}$, and $\tau_{3}$ were found to change. ${ }^{46}$ As one of these domains is converted to another, the magnetic easy plane (the plane of the cycloid) rotates, see Figure 7c. As illustrated in the same figure, the effect is reversible. This effect makes possible stabilisation of a single cycloidal magnetic domain in the entire macroscopic sample. Polarised neutron diffraction has shown that the magnetic cycloid has a single chirality (rotation direction) in a ferroelectric monodomain crystal. ${ }^{46}$ This result is consistent with theoretical analysis showing that the chirality of the cycloid in BFO is defined by the direction of the electric polarisation. ${ }^{49}$ The mechanism responsible for the electric-field-induced change of the equivalent magnetic domain fractions is probably related to the mechanical strain due to the inverse piezoelectric effect. As the sample deforms, the magnetic couplings along the three vectors $\tau_{\mathrm{i}}$ are no longer the same (for the experimental direction of the field along the [001]), and one of the magnetic domains becomes preferred energetically. This hypothesis was confirmed in neutron diffraction experiments under uniaxial pressure producing a similar strain. ${ }^{50}$ As expected, magnetic domain switching occurs as the pressure is applied. Remarkably low pressures $(<100$ bar) and strains $\left(<10^{-4}\right)$ are needed for the magnetic domain switching. This observation is of high importance for interpretation of phenomena occurring under applied fields and with changing temperature, as similar strains can easily appear under those circumstances.

Novel control of magnetic order by an applied electric field has been demonstrated in BFO single crystals. First, the magnetic order propagation vectors and the magnetic easy planes can be rotated together with the electric polarisation. Or, second, the populations of the three equivalent cycloidal magnetic domains within a ferroelectric monodomain can also be controlled by the field or uniaxial pressure. Neutron diffraction was instrumental in demonstrating these effects due to its unique ability to directly measure the populations of all the structural and magnetic domains in situ.

Recent neutron studies of BFO single crystals have revealed several important features of the magnetic order. First, SANS measurements have demonstrated ${ }^{51}$ a small tilting of the spins out of the cycloidal plane which takes the form of a SDW, see Figure 8. The importance of this observation is related to the destruction of the cycloidal modulation in strained BFO films and, possibly, in appropriately chemically altered samples. ${ }^{40}$ In these systems, the SDW is expected to transform into a weak ferromagnetic component, ${ }^{52}$ which could be of prime importance
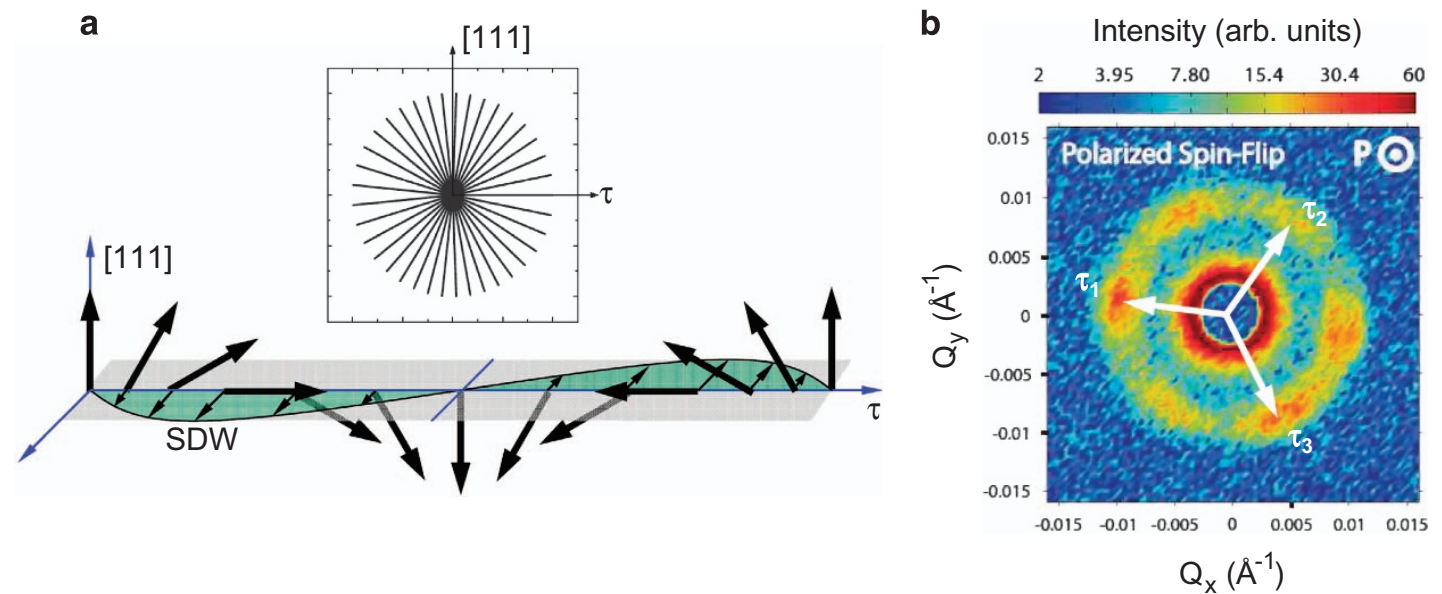

Figure 8. (a) The magnetic cycloid in $\mathrm{BiFeO}_{3}$. Only a few representative spins are shown, and the magnitude of the SDW is greatly exaggerated for clarity. The spin magnitude is $\sim 4 \mu_{B}$, and the SDW magnitude is $\sim 0.1 \mu_{B}$. There are $\sim 150$ spins in one period. The inset shows representative spins plotted using the same origin. A slight bunching along the [111] direction can be seen. (b) Polarised spin-flip SANS intensity observed on the two-dimensional position sensitive detector with the [111] direction normal to the image. $Q=0$ (incident beam direction) occurs in the center, and the magnitude of $Q$ increases with the distance away from $Q=0$. Six magnetic Bragg peaks due to the SDW in the $( \pm$ ) three equivalent domains are seen. In this geometry, the scattering is purely magnetic, and no signal due to the main (non-SDW) cycloidal component is present. (Adapted from reference 51). 
for applications given the rather large magnitude $\left(\sim 0.1 \mu_{B}\right)^{51}$ Second, observation of a weak third harmonic of the magnetic Bragg peaks has shown ${ }^{53}$ that the cycloid is not purely circular (the spins exhibit a slight bunching along the [111] direction), see Figure 8a. This 'anharmonicity' of the cycloid is important for interpretation of spectroscopic studies of BFO, since it affects the selection rules and allows assignment of previously mysterious modes. ${ }^{54}$ It also makes some of the excitation modes hybrid (combining magnetic and structural components), leading to the possibility of exotic effects, such as anomalous coupling to external fields. ${ }^{55}$ Temperature-dependent neutron diffraction studies have also been undertaken. ${ }^{53} \mathrm{~A}$ rather conventional behaviour of the magnetic order was found, and no spin-reorientation transitions were observed. This led to re-interpretation ${ }^{56}$ of the various bulk anomalies (in specific heat, electric susceptibility and so on) reported below room temperature for BFO. ${ }^{40}$

Recent synthesis of large single crystals has made possible inelastic neutron scattering studies of BFO. ${ }^{57-59}$ This allowed determination of the microscopic magnetic interaction parameters, more than 50 years since the discovery of this compound. The overall spin wave spectrum is well described by nearest and next-nearest neighbour AFM Heisenberg exchange constants of 4.38 and $0.15 \mathrm{meV}$, respectively. ${ }^{57}$ Studies of low-energy excitations indicate that the DM constant responsible for the cycloidal modulation is $\sim 0.1 \mathrm{meV}$, and the magnetic anisotropy energy producing the cycloid's anharmonicity is $\sim 10^{-2} \mathrm{meV}^{58}$ Subsequently, the DM coupling responsible for the SDW was found from Raman spectroscopy to be $\sim 0.05 \mathrm{meV}^{54}$ which is consistent with the observed SDW magnitude. ${ }^{51}$ These measurements provided a full description of the magnetic subsystem in $\mathrm{BFO}$, allowing for the first time detailed theoretical modelling with no fitting parameters. In particular, these results are of key importance for understanding of the infrared and Raman spectroscopies of BFO. ${ }^{54}$ Recently, anomalously large changes in the energetics of the low-energy spin waves (specifically, modes which correspond to oscillations of spins either within or outside of the cycloid plane) under applied electric field have been reported. ${ }^{60}$ These results could open new avenues for applications of multiferroics involving electric control of spin waves, especially in spintronic systems. Recent progress in crystal growth and in preparation of large monodomain BFO crystals finally makes possible neutron studies of the low-energy magnetic and hybrid excitations, including those in applied fields. This is an active current research field, with high potential for exciting results in the near future.

For device applications, typically multiferroic materials will be required in thin-film form. Spatial confinement of materials can alter the properties of materials compared with the bulk. For example, neutron scattering studies revealed that the long-range modulation observed in bulk BFO collapses to AFM G-type order in very thin BFO films. ${ }^{61}$ Studies of thicker films found modulation of the magnetic structure, although it was still different compared with the bulk. ${ }^{62,63}$ Further changes of the magnetic domain populations with application of an electric field were also observed with neutron scattering. ${ }^{64}$ These studies show the utility of neutron scattering for thin multiferroic films, such as those found in composite materials.

\section{COMPOSITE MATERIALS}

While the search for single-phase multiferroic materials exhibiting strong intrinsic magnetoelectric coupling continues, e.g., $\mathrm{EuTiO}_{3}$ (space group $P m \overline{3} m$ ), ${ }^{65}$ other approaches using nanoengineering to fabricate composite materials that exhibit magnetoelectric multiferroic behaviour as an extrinsic property, show promise.

Composite materials have long been important constituents of commercial products. By definition, composite materials contain interfaces, and with improvements in the synthesis and characterisation of materials at the nanoscale, the importance of a
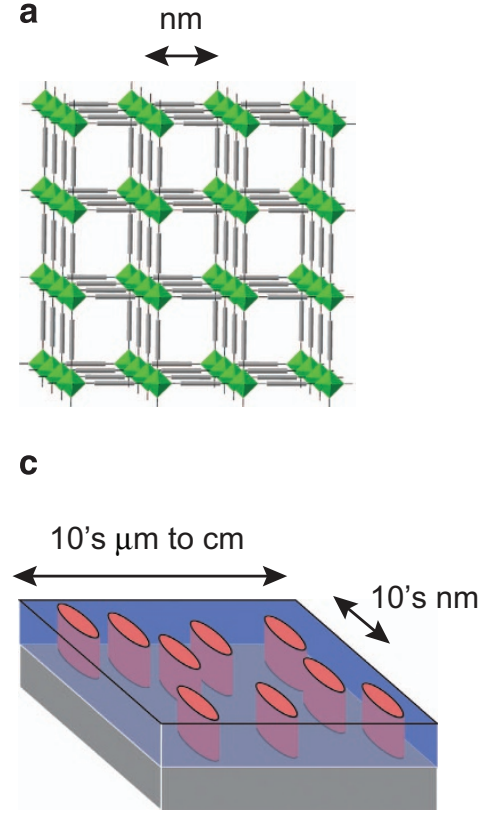

b

10 's $\mu \mathrm{m}$ to $\mathrm{cm}$

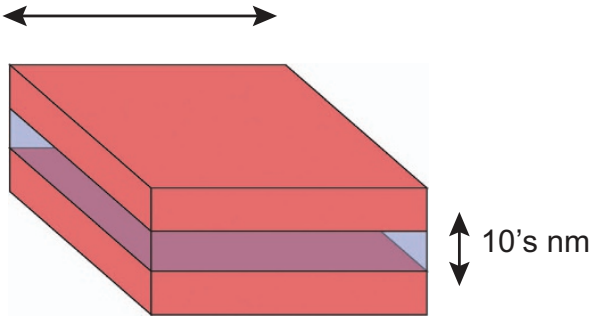

d

10 's $\mu \mathrm{m}$ to $\mathrm{cm}$

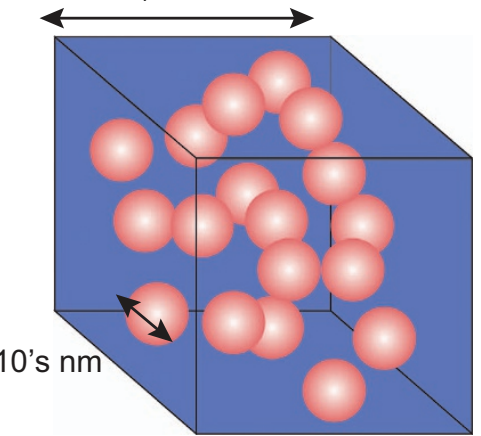

Figure 9. Concepts of magnetoelectric multiferroic nanocomposites. (a) A MOF of magnetic polyhedrons joined together by organic ligands (grey). In these systems the 'interface' consists of various bonds between the inorganic and organic components. (b-d) Magnetic component (red) in contact with a ferroelectric (blue) component. Interfacial regions can be increased by patterning (c) or use of nanocrystalline materials (d) compared to the thin film (planar) interface (b). Increased interfacial content may be one means of increasing magnetoelectric coupling. 
interfaces becomes preeminent. Nanoscale materials necessarily imply that interfaces play a decisive role in determining the response of composites to their environment. This is particularly true when interfaces mediate magnetoelectric coupling due to modification of spin-orbit coupling, e.g., as in rotation of oxygen octahedrons in a perovskite, ${ }^{66}$ hydrogen bonding in a metal organic framework (MOF), ${ }^{67}$ (spin) exchange coupling, ${ }^{3,68}$ or interfacial strain, ${ }^{69}$ and so on. A key requirement is to maximise the content of interfaces in the composite. The requirement can be realized in MOFs. Coupling may occur between the magnetic polyhedrons (green) and acentric organic building blocks (grey) taking place across numerous hydrogen and coordination bonds (Figure 9a). Couplings may also occur across interfaces as in bilayers or multilayers (Figure 9b), patterned materials (Figure 9c), and in two-phase bulk systems with nanometre-scale grains (Figure 9d). As coupling between different phases occurs across interfaces, characterisation of the structure, magnetic/electronic properties of interfaces and the influence of magnetic/electric fields and stress is paramount. Depending on the length scales of the interfaces, diffraction (discussed previously) or the specialized techniques of PNR and SANS are ideally suited to probe nuclear and magnetic structures over decades of length scales with nanometre resolution. Furthermore, sample environment can be constructed for neutron scattering to enable parametric studies of structure as functions of magnetic and electric fields, temperature and pressure.

\section{MAGNETISM OF PLANAR INTERFACES IN COMPOSITE MULTIFERROIC MATERIALS}

An often studied prototypical composite multiferroic system involves a thin-film ferromagnet (e.g., ( $\mathrm{La}, \mathrm{Sr}) \mathrm{MnO}_{3}$ (LSMO), CoFe and so on) grown on $\mathrm{BiFeO}_{3}$ (BFO). As described earlier, $\mathrm{BFO}$ is intrinsically a magnetoelectric multiferroic-exhibiting AFM and ferroelectric long-range order. However, by itself BFO is not technologically useful because it lacks the robust magnetisation of a ferromagnet which can be used to store information, produce spin currents and so on. To address this shortcoming, researchers have grown ferromagnetic films (e.g., LSMO, CoFe and so on) on BFO and vice versa. Interfacial coupling between the FM and the FE (BFO) across the interface produces the magnetoelectric coupling between magnetisation and polarisation. Indeed, the approach has met with some success; though, the coupling needs to be strengthened for technological applications. However, the mechanism of coupling is still unclear. In the case of LSMO/BFO heterostructures, distortions of oxygen octahedrons ${ }^{66}$ or epitaxial strain $^{69}$ may play a role. In the case of CoFe, a material with no magnetostriction, strain is not likely to be important. Here, coupling between the FM and the FE may occur due to a DM interaction that produces a canted AFM structure in the FE (which then couples to the FM) or exchange coupling across the interface between the $\mathrm{FM}$ and $\mathrm{FE}^{68,5}$ Indeed, a net uncompensated magnetisation in BFO ranging from 75 to $144 \mathrm{kA} / \mathrm{m}$ within a $\mathrm{nm}$ or so of the FM/BFO was reported from PNR studies for

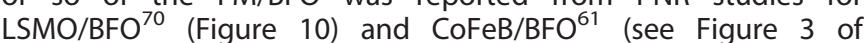
reference 60). Curiously, exchange bias, which is consistent with unidirectional anisotropy and DM interactions, was observed in $\mathrm{CoFeB} / \mathrm{BFO}^{61}$ but was absent in CoFe/BFO. ${ }^{68}$ Thus, further studies of these and other systems are required to clarify the mechanism of magnetoelectric coupling in FM/FE heterostructures. For example, the locations of pinned and unpinned uncompensated magnetisation in multiferroic heterostructures can be identified with PNR in the same way the technique was applied to studies of FM/AFM heterostructures. ${ }^{71,72}$

In some systems, strain can mediate or enhance magnetoelectric coupling. For example, epitaxial strain from a $\mathrm{DySCO}_{3}$ (space group $\mathrm{Pbnm}$ ) substrate stabilizes ferroelectricity and ferromagnetism simultaneously in a thin film of $\mathrm{EuTiO}_{3}$ when
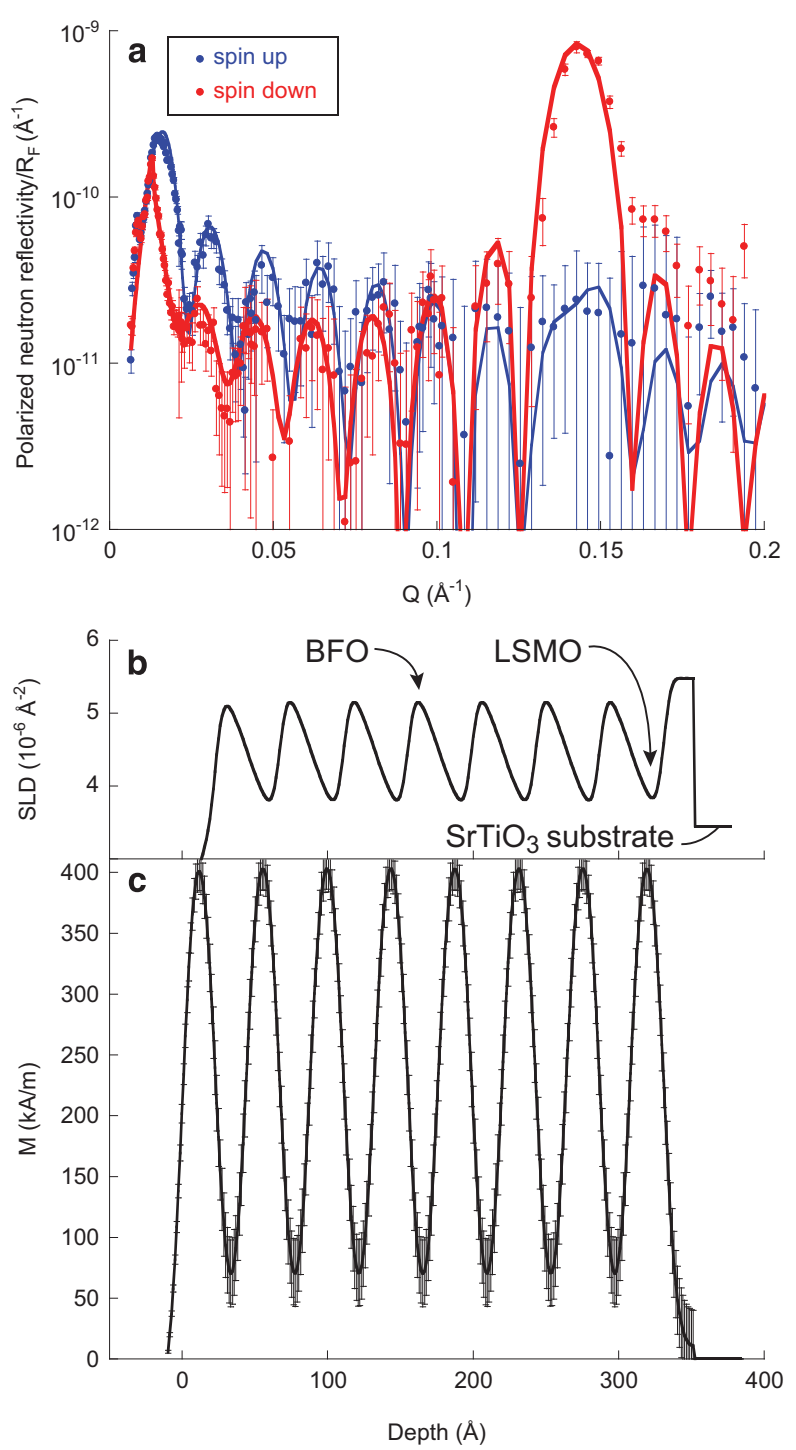

Figure 10. (a) Polarised neutron reflectivity (normalised to the Fresnel reflectivity $R_{\mathrm{F}}=16 \pi^{2} / Q^{4}$ ) of a BFO/LSMO superlattice at $10 \mathrm{~K}$ in an applied field of $0.1 \mathrm{~T}$. (b) Nuclear SLD and (c) magnetization depth profiles inferred from the data in a. The fitted spin-dependent reflectivities are the solid curves in a. Figure adapted from reference 70 .

neither property is present in the bulk. ${ }^{65}$ Recently, Singh et al. ${ }^{73}$ have been successful in inferring the magnetisation depth profile across interfaces using PNR measured as simultaneous functions of applied magnetic field, stress and temperature. Elastic bending stress was applied to the thin film using a four-point mechanical jig. Use of the new capability for studies of strain mediated magnetoelectric multiferroic heterostructures is an obvious next step, as is development of pressure cells for neutron reflectometry.

\section{MAGNETISM OF INTERFACES IN NANOCOMPOSITE} MULTIFERROIC BULK MATERIALS

Even with some of the promising developments recently realized, the magnetoelectric coupling of (planar) composite multiferroics remains small. Recognising that the interface is responsible for the coupling, some researchers have proposed to increase the interfacial component. This can be accomplished through lithography or by self-assembly of one component, or by mixing immiscible solutions so the resulting composite is chemically and 
magnetically non-uniform at length scales of tens of nm. Probing magnetic structures on such length scales with $\mathrm{nm}$ resolution, and especially in systems in which the interfaces are buried, is extraordinarily challenging. Fortunately, the techniques of grazing incidence SANS (GISANS, i.e., SANS in grazing incident angle reflection geometry), conventional SANS, and the especially powerful technique of SANS with polarised neutron beams and polarisation analysis of the scattering are ideal characterisation tools for these problems. SANS has been effective for identifying the correlation of magnetic lengths in materials exhibiting magnetic and electronic phase separation, 4,74 magnetisation reversal mechanisms of $\mathrm{nm}$-sized domains and domain walls in exchange bias systems, ${ }^{75}$ and the existence of uncompensated magnetisation in single crystals of $\mathrm{HoMnO}_{3} .{ }^{76}$ Notably, in the $\mathrm{HoMnO}_{3}$ study, control of the magnetisation via AFM domain walls as opposed to bulk magnetic order was demonstrated by application of an electric field during the SANS experiment. SANS has also identified differences of magnetic structure for nanoparticles and their interfaces in bulk materials. ${ }^{13}$ An important question SANS can answer is whether the induced magnetisation seen in the planar heterostructures (as depicted in Figure 9b, and reported in references 61,70 ) is also present in three-dimensional composite multiferroics (as depicted in Figure 9a,d).

\section{MULTIFERROIC HYBRID INORGANIC-ORGANIC MATERIALS}

Hybrid inorganic-organic materials are one of the major growth areas of materials science research. In particular, in the past 10 years, substantial effort has been devoted to three-dimensional nano-porous materials, also called MOFs. This has been primarily fuelled by record breaking surface areas reported for MOFs and concomitant utilisation for hydrogen gas storage, $\mathrm{CO}_{2}$ sequestration and hydrocarbon separation. However, recently researchers have utilised the dual nature of MOFs, namely inorganic and organic components, to realise functionalities otherwise not accessible in either category of materials. For example, Jain et al. ${ }^{67}$ reported multiferroic behaviour in $\left(\mathrm{CH}_{3}\right)_{3} \mathrm{NH}_{2} \mathrm{M}(\mathrm{HCOO})_{3}$ $(\mathrm{M}=\mathrm{Mn}, \mathrm{Co}, \mathrm{Ni}$ and $\mathrm{Fe}$ ) with two separate mechanisms leading to ferromagnetic and ferroelectric ordering. However, due to the complex twinning below the ferroelectric phase transition associated with hydrogen bond ordering and lack of neutron data, the low temperature ferroelectric structure in this prototypical multiferroic MOF remains elusive.

As the name suggests, MOFs can be thought of as composite materials made up of inorganic and organic building blocks. Metals are bound in a solid by coordination bonds to organic ligands with carboxylate or other functional groups. These framework materials are held together by any combination of coordination, hydrogen bonding and van der Waals forces. One can imagine an infinite number of such materials with varying topologies. The word 'Framework' in these materials is related to the cooperative interaction of valence electrons of neighbouring atoms. A consequence of these interactions is a wide range of cooperative phenomena such as ferroelectricity, magnetism and optical properties.

Studies of multiferroic MOFs represent a relatively new field and few examples of such materials are known to date. Earlier work on multiferroic MOFs utilised ordering of guest molecules in pores. Cui et al. ${ }^{77-81}$ reported a number of porous framework materials which exhibit a dielectric anomaly associated with the 'melting'(or 'freezing') of the positional freedom of trapped guest molecules. For example, $\left[\mathrm{Mn}_{3}(\mathrm{HCOO})_{6}\right]\left(\mathrm{C}_{2} \mathrm{H}_{5} \mathrm{OH}\right)$ exhibits ferroelectric and ferrimagnetic phase-transitions at 165 and $8.5 \mathrm{~K}$, respectively. The ferrimagnetic properties of this compound are due to the $\mathrm{Mn}^{2+}$ ions in the host lattice and the ferroelectric properties originate from the guest ethanol molecules. However, the volatility of the material is not conducive for technological applications.
In contrast, multiferroic behaviour reported in $\left(\mathrm{CH}_{3}\right)_{3} \mathrm{NH}_{2} \mathrm{M}$ $(\mathrm{HCOO})_{3}\left(\mathrm{M}=\mathrm{Mn}, \mathrm{Co}, \mathrm{Ni}\right.$ or Fe, which has the perovskite-like $\mathrm{ABX}_{3}$ topology, originates from the ordering of dimethyl ammonium cations (A site) -an integral part of the framework required for charge balancing-at the center of the $\mathrm{ReO}_{3}$ type cavity. ${ }^{67}$ Neutron diffraction studies have been carried out to shed light on the order-disorder phase transition observed in the mixed-valence $\mathrm{Fe}^{2+}-\mathrm{Fe}^{3+}$ formate framework compound $\left[\left(\mathrm{CH}_{3}\right)_{2}\right.$ $\left.\mathrm{NH}_{2}\right]_{n}\left[\mathrm{Fe}^{3+} \mathrm{Fe}^{2+}(\mathrm{HCOO})_{6}\right]_{n}{ }^{82}$ A combination of powder and single-crystal neutron diffraction measurements below the magnetic order transition $(\sim 37 \mathrm{~K})$ have been used to determine unequivocally the magnetic structure of this ferrimagnet. The ferrimagnetic moment, which arises as a result of the noncompensation of the $\mathrm{Fe}^{2+}-\mathrm{Fe}^{3+}$ sublattices, is calculated as the vector sum of the magnetic moments obtained from the neutron measurement at $2 \mathrm{~K}$ along the $c$ axis, $\sim 0.3 \mu_{\mathrm{B}}$. The spin moments of the $\mathrm{Fe}(6 b)$ and $\mathrm{Fe}(12 \mathrm{c})$ atoms lie strictly along the $c$ axis, while for those at the $18 e$ position, the moment has a main component along the $c$ axis which is coupled antiferromagnetically with the spin moments of the $6 b$ and $12 c$ sites. Together with a small component in the $a b$-plane a rotation of the magnetic moments materializes along the $c$ axis. $^{82}$ This duality of low and high spin states is a convenient means to establish a robust net magnetisation, required for technological applications.

A number of other multiferroic MOFs realized by replacing the dimethylammonium cation with hydroxyl ammonium and ammonium cations have also been reported. ${ }^{83,84}$ However, replacement of the dimethylammonium cation with guandidinium, $\mathrm{C}\left(\mathrm{NH}_{2}\right)_{3}^{+}$, in the copper formate analogue leads to an ordered polar structure at room temperature due to Jahn-Teller distortions. In this $\mathrm{Cu}$ MOF, Jahn-Teller and antiferro-distortions cooperate to induce a switchable ferroelectric polarisation by coupling to the A-site atoms through hydrogen bonding. Most importantly, A. Stroppa et al. ${ }^{85}$ show that a weak ferromagnetic component is coupled to the spontaneous polarisation, and they are mutually reversible.

Several molecular coordination materials are also candidate materials for magnetoelectric hybrid materials. Ferroelectric and magnetic phase transitions take place at almost the same temperature $(\sim 360 \mathrm{~K})$ in triethylmethylammonium tetrabromoferrate $(3+)$, resulting in strong magnetodielectric coupling. ${ }^{86} \mathrm{CuCl}_{4}\left(\mathrm{C}_{6} \mathrm{H}_{5} \mathrm{CH}_{2} \mathrm{CH}_{2} \mathrm{NH}_{3}\right)_{2}$, a molecular framework material with perovskite topology, shows spontaneous ferroelectric ordering below $340 \mathrm{~K}$, which coexists with ferromagnetic ordering below $13 \mathrm{~K}^{87}$ In this material, the electric polarisation results from the spatial ordering of hydrogen bonds that link the organic cations to the inorganic copper chloride block. Buckling of the corner-linked copper chloride octahedra drives hydrogen bond ordering. Because the magnetic exchange pathways are also determined by this octahedral buckling, a potentially large magnetoelectric coupling could be possible in this material. These molecular materials could easily rival prototypical oxide magnetoelectric materials and require further study.

Although, neutron scattering has often been applied to understand the magnetism of MOF materials, SANS has rarely been applied. ${ }^{88}$ Given the rich variety of modifications possible in this class of MOFs, for example, by alteration of organic ligand, template, strain and recent success in making thin films, new avenues are available to explore in the exciting field of multiferroic MOFs. Neutron scattering shall prove invaluable, as they have in oxides, in deciphering complex mechanisms in these complex hybrid materials.

\section{SUMMARY AND FUTURE DIRECTIONS}

Studies of multiferroic phenomena have been driven by novel materials. For potential device applications, there is a continued push to develop materials with magnetic transitions above room temperature. Presently, type II multiferroics with strong coupling 
between ferroelectric and magnetic order parameters appear very promising. Theory has been playing an increasing role in predicting new materials ${ }^{89}$ with strong coupling between ferroelectric and magnetic order parameters. Neutron scattering will continue to play a critical role in determining the magnetic structures of these materials and the strength of their exchange interactions, and how they couple to the electric polarisation. Furthermore, as more materials are studied in thin films and composites, neutron scattering will play an increasing role in determining the magnetic structure of these materials. In some devices where ferromagnetic layers are coupled to underlying multiferroic layers, neutron reflectivity measurements will be critical in determining the nature of the magnetic interactions between the multiferroic and ferromagnetic layers or composites of ferroelectric and ferromagnetic layers that exhibit multiferroic behaviour.

\section{ACKNOWLEDGEMENTS}

We thank our coauthors with whom we have worked, and Michel Kenzelmann and Owen P. Vajk for their help in preparing this review. We note that there have been a great many neutron studies of multiferroics, and in this brief overview only prototypical examples can be discussed, and with apologies to all the many other authors in this field, these are taken from our own work for convenience. V.K. was supported by DOE under Grant No. DE-FG02-07ER46382. Dr P.J. and Dr M.F. were supported by DOE Grant No. DE-AC52-06NA25396. Dr W.R. and Dr J.L. were supported by the Department of Commerce.

\section{COMPETING INTERESTS}

The authors declare no conflict of interest.

\section{REFERENCES}

1. Lynn, J. W. in Methods in Materials Research Vol. 3 (ed. Kaufmann, E. N.) 2253 (John Wiley \& Sons, 2012).

2. Lynn, J. W. Magnetic neutron scattering (invited). J. Appl. Phys. 75, 6806 (1994).

3. Lovesey, S. The Theory of Neutron Scattering from Condensed Matter (Clarendon Press, 1984).

4. Chatterji, T. Neutron Scattering from Magnetic Materials (Elsevier, 2006).

5. Izyumov, Y., Naish, V. E. \& Ozerov, R. P. Neutron Diffraction of Magnetic Materials (Springer, 1991).

6. Garcia, M. A. et al. Sources of experimental errors in the observation of nanoscale magnetism. J. Appl. Phys. 105, 013925 (2009).

7. Felcher, G. P. et al. Polarized neutron reflectometer: a new instrument to measure magnetic depth profiles. Rev. Sci. Instrum. 58, 609-619 (1987).

8. Majkrzak, C. F. Neutron scattering studies of magnetic thin films and multilayers. Phys. B Condens. Matter 221, 342-356 (1996).

9. Fitzsimmons, M. R. \& Majkrzak, C. F. in Modern Techniques for Characterizing Magnetic Materials 107-155 (Springer, 2005).

10. Gentile, T. R. et al. SANS polarization analysis with nuclear spin-polarized $3 \mathrm{He}$. J. Appl. Crystallogr. 33, 771-774 (2000).

11. Chen, W. C., Borchers, J. A., Erwin, R., Gentile, T. R. \& Lynn, J. W. Polarized $3 \mathrm{He}$ neutron spin filters for NCNR instruments. Notizario Neutroni E Luce di Sincrotrone 12, 32 (2007)

12. Chen, W. C. et al. Applications of 3 He neutron spin filters at the NCNR. Phys. $B$ Condens. Matter 404, 2663-2666 (2009).

13. Krycka, K. L. et al. Core-shell magnetic morphology of structurally uniform magnetite nanoparticles. Phys. Rev. Lett. 104, 207203 (2010).

14. Chae, S. C. et al. Direct observation of the proliferation of ferroelectric loop domains and vortex-antivortex pairs. Phys. Rev. Lett. 108, 167603 (2012).

15. Lorenz, B., Litvinchuk, A. P., Gospodinov, M. M. \& Chu, C. W. Field-induced reentrant novel phase and a ferroelectric-magnetic order coupling in $\mathrm{HoMnO}_{3}$. Phys. Rev. Lett. 92, 087204-087204 (2004).

16. Lottermoser, T. et al. Magnetic phase control by an electric field. Nature $\mathbf{4 3 0}$, 541-544 (2004)

17. Vajk, O. P., Kenzelmann, M., Lynn, J. W., Kim, S. B. \& Cheong, S. W. Magnetic order and spin dynamics in ferroelectric $\mathrm{HoMnO}_{3}$. Phys. Rev. Lett. 94, 087601 (2005).

18. Choi, Y. J. et al. Giant magnetic fluctuations at the critical endpoint in insulating $\mathrm{HoMnO}_{3}$. Phys. Rev. Lett. 110, 157202 (2013).

19. Vajk, O. P., Kenzelmann, M., Lynn, J. W., Kim, S. B. \& Cheong, S. W. Neutron-scattering studies of magnetism in multiferroic $\mathrm{HoMnO}_{3}$ (invited). J. Appl. Phys. 99, $08 \mathrm{E} 301$ (2006).
20. Skanthakumar, S. et al. Magnetic phase transitions and structural distortion in $\mathrm{Nd}_{2} \mathrm{CuO}_{4}$. Phys. C: Supercond. 160, 124-128 (1989).

21. Kimura, T. et al. Distorted perovskite with $e_{g}^{1}$ configuration as a frustrated spin system. Phys. Rev. B 68, 060403 (2003).

22. Kimura, T. et al. Magnetic control of ferroelectric polarization. Nature 426, 55-58 (2003).

23. Quezel, S., Tcheou, F., Rossat-Mignod, J., Quezel, G. \& Roudaut, E. Magnetic structure of the perovskite-like compound $\mathrm{TbMnO}_{3}$. Physica $B+C$ 86-88, 916-918 (1977).

24. Kenzelmann, M. et al. Magnetic inversion symmetry breaking and ferroelectricity in $\mathrm{TbMnO}_{3}$. Phys. Rev. Lett. 95, 087206 (2005).

25. Wilkins, S. B. et al. Nature of the magnetic order and origin of induced ferroelectricity in $\mathrm{TbMnO}_{3}$. Phys. Rev. Lett. 103, 207602 (2009).

26. Terada, N. et al. Magnetic ordering in pressure-induced phases with giant spindriven ferroelectricity in multiferroic $\mathrm{TbMnO}_{3}$. Phys. Rev. B 93, 081104 (2016).

27. Stein, J. et al. Control of multiferroic domains by external electric fields in $\mathrm{TbMnO}_{3}$. J. Phys. Condens. Matter 446001, 446001 (2015).

28. Mostovoy, M. Ferroelectricity in spiral magnets. Phys. Rev. Lett. 96, 067601 (2006)

29. Katsura, H., Nagaosa, N. \& Balatsky, A. V. Spin current and magnetoelectric effect in noncollinear magnets. Phys. Rev. Lett. 95, 057205 (2005).

30. Sergienko, I. A. \& Dagotto, E. Role of the Dzyaloshinskii-Moriya interaction in multiferroic perovskites. Phys. Rev. B 73, 094434 (2006).

31. $\mathrm{Hu}, \mathrm{J}$. Microscopic origin of magnetoelectric coupling in noncollinear multiferroics. Phys. Rev. Lett. 100, 077202 (2008).

32. Meddar, L. et al. Effect of nonmagnetic substituents $\mathrm{Mg}$ and $\mathrm{Zn}$ on the phase competition in the multiferroic antiferromagnet $\mathrm{MnWO}_{4}$. Chem. Mater. 21 5203-5214 (2009).

33. Malashevich, A. \& Vanderbilt, D. Dependence of electronic polarization on octahedral rotations in $\mathrm{TbMnO}_{3}$ from first principles. Phys. Rev. B 80, 224407 (2009).

34. Senff, D. et al. Magnetic excitations in multiferroic $\mathrm{TbMnO}_{3}$ : evidence for a hybridized soft mode. Phys. Rev. Lett. 98, 137206 (2007).

35. Senff, D. et al. Magnetic excitations in a cycloidal magnet: the magnon spectrum of multiferroic $\mathrm{TbMnO}_{3}$. J. Phys. Condens. Matter 20, 434212 (2008).

36. Katsura, H., Balatsky, A. V. \& Nagaosa, N. Dynamical magnetoelectric coupling in helical magnets. Phys. Rev. Lett. 98, 027203 (2007).

37. Valdés Aguilar, R. et al. Origin of electromagnon excitations in multiferroic $\mathrm{RMnO}_{3}$. Phys. Rev. Lett. 102, 047203 (2009).

38. Cabrera, I. et al. Coupled magnetic and ferroelectric domains in multiferroic $\mathrm{Ni}_{3} \mathrm{~V}_{2} \mathrm{O}_{8}$. Phys. Rev. Lett. 103, 087201 (2009).

39. Senff, D., Link, P., Aliouane, N., Argyriou, D. N. \& Braden, M. Field dependence of magnetic correlations through the polarization flop transition in multiferroic $\mathrm{TbMnO}_{3}$ : Evidence for a magnetic memory effect. Phys. Rev. B 77, 174419 (2008).

40. Catalan, G. \& Scott, J. F. Physics and applications of bismuth ferrite. Adv. Mater. 21, 2463-2485 (2009).

41. Royen, P., Swars, K. Das System Wismutoxyd-Eisenoxyd im Bereich von 0 bis 55 Mol\% Eisenoxyd. Angewandte Chemie 69, 779 (1957).

42. Lebeugle, D. et al. Room-temperature coexistence of large electric polarization and magnetic order in $\mathrm{BiFeO}_{3}$ single crystals. Phys. Rev. B 76, 024116 (2007).

43. Lee, S., Ratcliff, W., Cheong, S. \& Kiryukhin, V. Electric field control of the magnetic state in $\mathrm{BiFeO}_{3}$ single crystals. Appl. Phys. Lett. 92, 192906 (2008).

44. Sosnowska, I., Neumaier, T. P. \& Steichele, E. Spiral magnetic ordering in bismuth ferrite. J. Phys. C Solid State Phys. 15, 4835-4846 (1982).

45. Lebeugle, D. et al. Electric-field-induced spin-flop in $\mathrm{BiFeO}_{3}$ single crystals at room-temperature. Phys. Rev. Lett. 227602, 1-4 (2008).

46. Lee, S. et al. Single ferroelectric and chiral magnetic domain of single-crystalline $\mathrm{BiFeO}_{3}$ in an electric field. Phys. Rev. B 78, 100101 (2008).

47. Park, J. et al. Magnetoelectric feedback among magnetic order, polarization, and lattice in multiferroic $\mathrm{BiFeO}_{3}$. J. Phys. Soc. Jpn 80, 114714 (2011).

48. Chu, Y.-H. et al. Electric-field control of local ferromagnetism using a magnetoelectric multiferroic. Nat. Mater. 7, 478-482 (2008).

49. Kadomtseva, A. M. et al. Space-time parity violation and magnetoelectric interactions in antiferromagnets. JETP Lett. 79, 571-581 (2004).

50. Ramazanoglu, M. et al. Giant effect of uniaxial pressure on magnetic domain populations in multiferroic bismuth ferrite. Phys. Rev. Lett. 107, 067203 (2011).

51. Ramazanoglu, M. et al. Local weak ferromagnetism in single-crystalline ferroelectric $\mathrm{BiFeO}_{3}$. Phys. Rev. Lett. 107, 207206 (2011).

52. Ederer, C. \& Spaldin, N. A. Weak ferromagnetism and magnetoelectric coupling in bismuth ferrite. Phys. Rev. B 71, 060401 (2005).

53. Ramazanoglu, M. et al. Temperature-dependent properties of the magnetic order in single-crystal $\mathrm{BiFeO}_{3}$. Phys. Rev. B 83, 174434 (2011).

54. Fishman, R. S., Haraldsen, J. T., Furukawa, N. \& Miyahara, S. Spin state and spectroscopic modes of multiferroic $\mathrm{BiFeO}_{3}$. Phys. Rev. B 87, 134416 (2013).

55. de Sousa, R. \& Moore, J. E. Optical coupling to spin waves in the cycloidal multiferroic $\mathrm{BiFeO}_{3}$. Phys. Rev. B 77, 012406 (2008). 
56. Jarrier, R. et al. Surface phase transitions in $\mathrm{BiFeO}_{3}$ below room temperature. Phys. Rev. B 85, 184104 (2012).

57. Jeong, J. et al. Spin wave measurements over the full Brillouin zone of multiferroic $\mathrm{BiFeO}_{3}$. Phys. Rev. Lett. 108, 077202 (2012).

58. Matsuda, M. et al. Magnetic dispersion and anisotropy in multiferroic $\mathrm{BiFeO}_{3}$. Phys. Rev. Lett. 109, 067205 (2012).

59. Xu, Z. et al. Thermal evolution of the full three-dimensional magnetic excitations in the multiferroic $\mathrm{BiFeO}_{3}$. Phys. Rev. B 86, 174419 (2012).

60. Rovillain, P. et al. Electric-field control of spin waves at room temperature in multiferroic $\mathrm{BiFeO}_{3}$. Nat. Mater. 9, 975-979 (2010).

61. Béa, $\mathrm{H}$. et al. Mechanisms of exchange bias with multiferroic $\mathrm{BiFeO}_{3}$ epitaxial thin films. Phys. Rev. Lett. 100, 017204 (2008).

62. Ratcliff, W. et al. Neutron diffraction investigations of magnetism in $\mathrm{BiFeO}_{3}$ epitaxial films. Adv. Funct. Mater. 21, 1567-1574 (2011).

63. Ke, X. et al. Magnetic structure of epitaxial multiferroic $\mathrm{BiFeO}_{3}$ films with engineered ferroelectric domains. Phys. Rev. B 82, 134448 (2010).

64. Ratcliff, W. et al. Electric-field-controlled antiferromagnetic domains in epitaxial $\mathrm{BiFeO}_{3}$ thin films probed by neutron diffraction. Phys. Rev. B 87, 140405 (2013).

65. Lee, J. H. et al. A strong ferroelectric ferromagnet created by means of spin-lattice coupling. Nature 466, 954-958 (2010).

66. Rondinelli, J. M., May, S. J. \& Freeland, J. W. Control of octahedral connectivity in perovskite oxide heterostructures: an emerging route to multifunctional materials discovery. MRS Bull. 37, 261-270 (2012).

67. Jain, P. et al. Multiferroic behavior associated with an order-disorder hydrogen bonding transition in metal-organic frameworks (MOFs) with the perovskite $\mathrm{ABX}_{3}$ architecture. J. Am. Chem. Soc. 131, 13625-13627 (2009).

68. Trassin, M. et al. Interfacial coupling in multiferroic/ferromagnet heterostructures. Phys. Rev. B 87, 134426 (2013).

69. Eerenstein, W., Mathur, N. D. \& Scott, J. F. Multiferroic and magnetoelectric materials. Nature 442, 759-765 (2006).

70. Singh, S. et al. Induced magnetization in $\mathrm{La}_{0.7} \mathrm{Sr}_{0.3} \mathrm{MnO}_{3} / \mathrm{BiFeO}_{3}$ superlattices. Phys. Rev. Lett. 113, 047204 (2014).

71. Fitzsimmons, M. et al. Asymmetric magnetization reversal in exchange-biased hysteresis loops. Phys. Rev. Lett. 84, 3986-3989 (2000).

72. Fitzsimmons, M. R. et al. Pinned magnetization in the antiferromagnet and ferromagnet of an exchange bias system. Phys. Rev. B 75, 214412 (2007).

73. Singh, S. et al. Role of elastic bending stress on magnetism of a manganite thin film studied by polarized neutron reflectometry. Phys. Rev. B 85, 214440 (2012)

74. Torija, M. A. et al. Chemically driven nanoscopic magnetic phase separation at the $\mathrm{SrTiO}_{3}(001) / \mathrm{La}_{1-x} \mathrm{Sr}_{x} \mathrm{CoO}_{3}$ interface. Adv. Mater. 23, 2711-2715 (2011).

75. Dufour, C. et al. Nanometer-size magnetic domains and coherent magnetization reversal in a giant exchange-bias system. Phys. Rev. B 84, 064420 (2011).

76. Ueland, B. G., Lynn, J. W., Laver, M., Choi, Y. J. \& Cheong, S.-W. Origin of electric-field-induced magnetization in multiferroic $\mathrm{HoMnO}_{3}$. Phys. Rev. Lett. 104, 147204 (2010).
77. Cui, H.-B. et al. Dielectric properties of porous molecular crystals that contain polar molecules. Angew. Chem. 117, 6666-6670 (2005).

78. Cui, H.-B. et al. Dielectric properties of porous molecular crystals that contain polar molecules. Angew. Chem. Int. Ed. Engl. 44, 6508-6512 (2005).

79. Cui, H. et al. Ferroelectric porous molecular crystal, $\left[\mathrm{Mn}_{3}(\mathrm{HCOO})_{6}\right]\left(\mathrm{C}_{2} \mathrm{H}_{5} \mathrm{OH}\right)$, exhibiting ferrimagnetic transition. J. Am. Chem. Soc. 128, 15074-15075 (2006).

80. Cui, H. et al. A porous coordination-polymer crystal containing one-dimensional water chains exhibits guest-induced lattice distortion and a dielectric anomaly. Angew. Chem. Int. Ed. Engl. 47, 3376-3380 (2008).

81. Zhou, B. et al. Anomalous dielectric behavior and thermal motion of water molecules confined in channels of porous coordination polymer crystals. J. Am. Chem. Soc. 133, 5736-5739 (2011).

82. Cañadillas-Delgado, L. et al. The role of order-disorder transitions in the quest for molecular multiferroics: structural and magnetic neutron studies of a mixed valence iron(II)-iron(III) formate framework. J. Am. Chem. Soc. 134, 19772-19781 (2012).

83. Liu, B., Shang, R., Hu, K.-L., Wang, Z.-M. \& Gao, S. A new series of chiral metal formate frameworks of $\left[\mathrm{HONH}_{3}\right]\left[\mathrm{M}(\mathrm{II})(\mathrm{HCOO})_{3}\right](\mathrm{M}=\mathrm{Mn}, \mathrm{Co}, \mathrm{Ni}, \mathrm{Zn}$, and $\mathrm{Mg})$ : synthesis, structures, and properties. Inorg. Chem. 51, 13363-13372 (2012).

84. $\mathrm{Xu}, \mathrm{G} .-\mathrm{C}$. et al. Coexistence of magnetic and electric orderings in the metalformate frameworks of $\left[\mathrm{NH}_{4}\right]\left[\mathrm{M}(\mathrm{HCOO})_{3}\right]$. J. Am. Chem. Soc. 133, 14948-14951 (2011).

85. Stroppa, A. et al. Electric control of magnetization and interplay between orbital ordering and ferroelectricity in a multiferroic metal-organic framework. Angew. Chem. Int. Ed. Engl. 50, 5847-5850 (2011).

86. Cai, H.-L. et al. Above-room-temperature magnetodielectric coupling in a possible molecule-based multiferroic: triethylmethylammonium tetrabromoferrate(III). J. Am. Chem. Soc. 134, 18487-18490 (2012).

87. Polyakov, A. O. et al. Coexisting ferromagnetic and ferroelectric order in a $\mathrm{CuCl}_{4}$ -based organic-inorganic hybrid. Chem. Mater. 24, 133-139 (2012).

88. Nayuk, R. et al. Modulated formation of MOF-5 nanoparticles-a SANS analysis. J. Phys. Chem. C 116, 6127-6135 (2012).

89. Fennie, C. J. \& Rabe, K. M. Magnetic and electric phase control in epitaxial $\mathrm{EuTiO}_{3}$ from first principles. Phys. Rev. Lett. 97, 267602 (2006).

This work is licensed under a Creative Commons Attribution 4.0 International License. The images or other third party material in this article are included in the article's Creative Commons license, unless indicated otherwise in the credit line; if the material is not included under the Creative Commons license, users will need to obtain permission from the license holder to reproduce the material. To view a copy of this license, visit http://creativecommons.org/licenses/ by/4.0/

(c) The Author(s) 2016 